\title{
Predictive value of perioperative near-infrared spectroscopy for neurodevelopmental outcomes after cardiac surgery in infancy
}

\author{
Erica D. Sood, PhD, ${ }^{\mathrm{a}, \mathrm{b}}$ Julie S. Benzaquen, $\mathrm{PhD},{ }^{\mathrm{a}, \mathrm{b}}$ Ryan R. Davies, MD, ${ }^{\mathrm{a}}$ Edward Woodford, MPAS, ${ }^{\mathrm{a}}$ and \\ Christian Pizarro, MD
}

Objective: The study objective was to expand on prior research examining intraoperative regional cerebral oxygen saturation $\left(\mathrm{rSO}_{2}\right)$ measured by near-infrared spectroscopy (NIRS) by evaluating the predictive value of perioperative NIRS monitoring for neurodevelopmental outcomes after infant cardiac surgery.

\begin{abstract}
Methods: Cross-sectional neurodevelopmental evaluation at 24 months of age with the Bayley Scales of Infant and Toddler Development, Third Edition was performed for patients who underwent cardiac surgery with perioperative NIRS monitoring between 2007 and 2010. Retrospective clinical data were extracted from the electronic medical record. Evaluation of selected NIRS measures, including preoperative $\mathrm{rSO}_{2}$ (baseline) as well as $\mathrm{rSO}_{2}$ nadir and percent decrease from baseline during the intraoperative and early postoperative periods, was undertaken.
\end{abstract}

Results: Perioperative NIRS and neurodevelopmental data were available for 31 patients without chromosomal anomalies who underwent cardiac surgery during infancy at a median age of 0.43 months. Optimal thresholds on NIRS measures identified through receiver operating characteristic analyses were intraoperative percent decrease of $52 \%$ for receptive communication delay and postoperative $\mathrm{rSO}_{2}$ nadir of $56 \%$ for cognitive delay and $49 \%$ for gross motor delay. When considered in conjunction with other clinical characteristics in stepwise linear regression analyses, intraoperative percent decrease of more than $52 \%$ entered into the final model for receptive communication outcome and postoperative $\mathrm{rSO}_{2}$ nadir of less than $56 \%$ entered into the final model for cognitive outcome.

Conclusions: Perioperative NIRS monitoring seems to enhance the ability to predict neurodevelopmental outcome. Specific NIRS measures associated with neurodevelopmental outcome, as well as optimal thresholds, seem to differ across the continuum of the perioperative period. (J Thorac Cardiovasc Surg 2013;145:438-45)

Supplemental material is available online.

With the dramatic decline in mortality for children with congenital heart disease (CHD), attention has been directed toward functional outcomes. Neurodevelopmental (ND) impairment is the most common morbidity affecting the quality of life of children with CHD who required surgical intervention. ${ }^{1}$ Perioperative regional cerebral oxygen

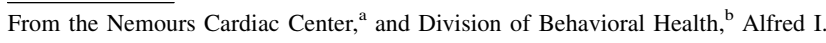
duPont Hospital for Children, Wilmington, Del.

This research was supported by a grant from the Nemours Foundation (Grant 16-07700-001) and also made possible through the generosity of donors (Nemours Fund for Children's Health).

Disclosures: Authors have nothing to disclose with regard to commercial support.

Read at the 92nd Annual Meeting of The American Association for Thoracic Surgery, San Francisco, California, April 28-May 2, 2012.

Received for publication April 26, 2012; revisions received Aug 29, 2012; accepted for publication Oct 22, 2012; available ahead of print Dec 7, 2012.

Address for reprints: Erica D. Sood, PhD, Nemours Cardiac Center and Division of Behavioral Health, Alfred I. duPont Hospital for Children, 1600 Rockland Rd, Wilmington, DE 19803 (E-mail: esood@nemours.org).

$0022-5223 / \$ 36.00$

Copyright (C) 2013 by The American Association for Thoracic Surgery http://dx.doi.org/10.1016/j.jtcvs.2012.10.033 saturation $\left(\mathrm{rSO}_{2}\right)$ has been identified as a potential contributor to ND outcomes in this population ${ }^{2,3}$; as a result, monitoring $\mathrm{rSO}_{2}$ with near-infrared spectroscopy (NIRS), a noninvasive optical technique that monitors brain tissue oxygen saturation through measurement of oxyhemoglobin and de-oxyhemoglobin concentrations, is gaining acceptance. ${ }^{4-6}$ However, a body of research correlating NIRS data with ND scores and showing that these data can be used to improve outcomes is needed before the value of routine NIRS monitoring for pediatric cardiac surgery can be determined. ${ }^{7,8}$

The few published studies that have examined the relationship between $\mathrm{rSO}_{2}$ measured by NIRS and ND outcomes have focused exclusively on the preoperative or intraoperative (IO) periods. ${ }^{9-11}$ Despite extensive research documenting decreased oxygen delivery during the early postoperative (PO) period ${ }^{12-15}$ and an association between PO events (eg, seizures, cardiac arrest, circulatory instability) and neurologic or ND outcomes, ${ }^{16-18}$ there are no published data examining the relationships between PO NIRS measures and neurodevelopment. Without these data, the predictive and clinical utility of this tool during the perioperative period are unknown. This study seeks to expand on our previous work on the assessment of IO 

Abbreviations and Acronyms
AUC = area under the curve
$\mathrm{CHD}=$ congenital heart disease
$\mathrm{CPB}=$ cardiopulmonary bypass
DHCA $=$ deep hypothermic circulatory arrest
IO = intraoperative
$\mathrm{ND}=$ neurodevelopmental
NIRS = near-infrared spectroscopy
$\mathrm{NPV}=$ negative predictive value
$\mathrm{PO}=$ postoperative
$\mathrm{PPV}=$ positive predictive value
$\mathrm{ROC}=$ receiver operating characteristic
$\mathrm{rSO}_{2}=$ regional cerebral oxygen saturation

$\mathrm{rSO}_{2}$ by evaluating the predictive value of selected NIRS measures across the continuum of the perioperative period for ND outcome at 24 months of age.

\section{MATERIALS AND METHODS \\ Participants and Study Design}

Participants were a subset $(\mathrm{N}=31)$ of 62 patients enrolled across 2 crosssectional, retrospective research studies examining ND outcome at 24 months of age after infant cardiac surgery. ${ }^{11,19}$ The Nemours Institutional Review Board approved both studies, and parental permission was obtained from the parents/legal guardians before participation. Among 44 patients without a chromosomal anomaly, 31 had perioperative NIRS data available for the first $(\mathrm{N}=29)$ or second $(\mathrm{N}=2)$ surgery with cardiopulmonary bypass (CPB) between 2007 and 2010. All patients were aged less than 12 months at the time of surgery and were aged $24 \pm 3$ months at the time of the ND assessment.

\section{Operative Management}

Patients were managed preoperatively and postoperatively in a dedicated cardiac intensive care unit. Operative management has been described. ${ }^{11}$ Perioperative management decisions were not made on the basis of NIRS data. The majority of patients in this sample had deep hypothermic circulatory arrest (DHCA) $(94 \%)$, because this was an inclusion criterion for one of the larger studies. Total body intermittent perfusion was performed when a period of DHCA greater than 40 minutes was anticipated.

\section{Near-Infrared Spectroscopy}

Recording of $\mathrm{rSO}_{2}$ with NIRS (INVOS 5100B; INVOS 5100C; Somanetics, Troy, Mich) was undertaken before, during, and up to 48 hours after congenital cardiac surgery by placement of a probe on the forehead in the midline position. The first NIRS value after induction of anesthesia was considered the baseline $\mathrm{rSO}_{2}$. NIRS readings during the IO and early PO periods were used to calculate the lowest $\mathrm{rSO}_{2}\left(\mathrm{rSO}_{2}\right.$ nadir $)$ and percent decrease from baseline $\mathrm{rSO}_{2}$ (percent decrease) across the continuum of the perioperative period and separately for the IO and PO periods.

\section{Neurodevelopmental Testing}

Children were administered the Bayley Scales of Infant and Toddler Development, Third Edition ${ }^{20}$ by a clinical psychologist. The Bayley Scales of Infant and Toddler Development, Third Edition assesses developmental functioning through items administered in a structured play format. Compared with the second edition of the Bayley Scales, this updated measure allows for the calculation of separate cognitive, receptive communication, and expressive communication scaled scores (previously combined into the Mental Development Index) and separate fine and gross motor scaled scores (previously combined into the Psychomotor Development Index). Scaled scores have a mean of 10 and a standard deviation of 3 . We obtained only partial ND data for 5 patients because of child fussiness or time constraints. All available data for these patients were included in analyses.

\section{Analytic Strategy}

Exploratory univariable analyses assessed the relationships of NIRS measures and sociodemographic and clinical variables to ND scores. Receiver operating characteristic (ROC) analyses were then conducted to evaluate whether NIRS measures could be used to determine risk for ND delay, and if so, to identify the optimal threshold for ruling in and out delay. Only those relationships with a $P$ value less than .10 in exploratory analyses were included in ROC analyses. Area under the curve (AUC) was reported as the measure of effect size, with 0.56 signifying the minimum for a small effect, 0.64 signifying the minimum for a medium effect, and 0.71 signifying the minimum for a large effect. ${ }^{21}$ For the purposes of ROC analyses, patients were stratified into 2 groups for each ND domain. Patients were considered delayed if their score was more than 1 standard deviation below the normative mean (scaled scores of 1-6). All other patients were considered nondelayed.

To evaluate whether NIRS measures make a significant contribution to the prediction of ND scores when considered in conjunction with other patient- and procedure-related variables, stepwise linear regression analyses were conducted. Dichotomous (greater than and less than identified thresholds) NIRS measures and sociodemographic and clinical characteristics significantly associated with ND scores in univariable analyses were considered for entry into multivariable models (see list in Table E1). Explained variance for all models was calculated by adjusted $R^{2}$ values. The Nemours Foundation was not involved in data interpretation.

\section{RESULTS}

\section{Clinical Characteristics}

Table 1 shows the clinical and sociodemographic characteristics of the 31 participants in this sample. Cardiac procedures consisted of the Norwood $(\mathrm{N}=6)$, ventricular septal defect repair ( \pm coarctation) $(\mathrm{N}=5)$, arch repair $(\mathrm{N}=3)$, arterial switch ( \pm ventricular septal defect repair) $(\mathrm{N}=3)$, hemi-Fontan $(\mathrm{N}=2)$, Ross-Konno ( \pm arch repair) $(\mathrm{N}=2)$, total anomalous pulmonary venous connection repair $(\mathrm{N}=2)$, double-outlet right ventricle repair $(\mathrm{N}=2)$, tetralogy of Fallot repair $(\mathrm{N}=2)$, orthotopic heart transplantation $(\mathrm{N}=1)$, truncus repair $(\mathrm{N}=1)$, hybrid II $(\mathrm{N}=1)$, and cor triatriatum repair $(\mathrm{N}=1)$. One patient had cardiac arrest and required extracorporeal membrane oxygenation before the 24-month ND assessment. Procedure-related characteristics referred to the first $(\mathrm{N}=29)$ or second $(\mathrm{N}=2)$ procedure with $\mathrm{CPB}$ for which perioperative NIRS data were available. Five patients underwent 1 additional cardiac procedure, 10 patients underwent 2 additional cardiac procedures, and 1 patient underwent 3 additional cardiac procedures before the 24-month ND assessment. Among these patients, the first procedure was typically the most complex intervention, often performed during the newborn period. Table 2 shows the perioperative NIRS data. Single ventricle physiology was associated with 
TABLE 1. Clinical and sociodemographic characteristics

\begin{tabular}{lc}
\hline Clinical characteristics & Median (1Q-3Q) \\
\hline Birth weight (kg) & $3.2(2.7-3.5)$ \\
Age at surgery (mo) & $0.4(0.1-2)$ \\
Weight at surgery (kg) & $3.6(3.2-4.3)$ \\
Aristotle complexity score & $13(10-15.5)$ \\
CPB support (min) & $106.5(75.8-131)$ \\
DHCA (min) & $42.0(35-50)$ \\
CICU stay (d) & $9(4-12)$ \\
Hospital stay (d) & $17(12-23)$ \\
Cumulative exposure to DHCA (min) & $45(35-80)$ \\
\hline Clinical characteristics & Frequency $(\%)$ \\
\hline Premature birth (<37 wk) & $3(10 \%)$ \\
Significant comorbidity* & $8(25.8 \%)$ \\
Single ventricle physiology & $10(32.3 \%)$ \\
Preoperative CICU & $21(67.7 \%)$ \\
Use of DHCA & $29(93.5 \%)$ \\
Preoperative cyanosis & $16(51.6 \%)$ \\
Multiple cardiac procedures (lifetime) & $16(51.6 \%)$ \\
Multiple procedures with DHCA (lifetime) & $11(35.4 \%)$ \\
\hline Sociodemographic characteristics & Frequency (\%) \\
\hline Race/ethnicity & \\
Non-Hispanic white & $24(77.4 \%)$ \\
Black or African-American & $5(16.1 \%)$ \\
Other & $2(6.4 \%)$ \\
Household income & \\
< $\$ 39,999$ & $8(25.8 \%)$ \\
\$40,000-\$69,999 & $6(19.4 \%)$ \\
\$70,000-\$99,999 & $7(22.6 \%)$ \\
$>\$ 100,000$ & $7(22.6 \%)$ \\
Missing & $3(9.7 \%)$ \\
Education level of accompanying parent & $15(50 \%)$ \\
Education level of spouse/partner & $16(57.1 \%)$ \\
College degree or higher & \\
\hline & \\
\hline &
\end{tabular}

$1 Q$, First quartile; $3 Q$, third quartile; $C P B$, cardiopulmonary bypass; $D H C A$, deep hypothermic circulatory arrest; $C I C U$, cardiac intensive care unit. *Significant comorbidities included prematurity less than 35 weeks gestational age, arrhythmia, necrotizing enterocolitis, depressed myocardial function, protein losing enteropathy/right ventricle dysfunction/pacemaker, and pancreatic insufficiency.

lower preoperative $\mathrm{rSO}_{2}(t[29]=-2.0, P=.05)$ and $\mathrm{PO}$ $\mathrm{rSO}_{2}$ nadir $(t[29]=-4.9, P<.001)$, and duration of DHCA was negatively associated with $\mathrm{IO} \mathrm{rSO}_{2}$ nadir $(r=-0.42, P=.02)$ and $\mathrm{PO} \mathrm{rSO}_{2}$ nadir $(r=-0.41$, $P=.02) . \mathrm{PO} \mathrm{rSO}_{2}$ nadir was significantly associated with PO lactate levels at 8 hours $(r=-0.40, P=.02)$, but not at $16(r=-0.24, P=.19)$ or $24(r=-0.14, P>.20)$ hours, when the majority of lactate levels were less than $2(84 \%$ and $79 \%$, respectively).

\section{Neurodevelopmental Outcomes}

Mean scores for the cognitive $(8.8 \pm 3.3)$, receptive communication $(9.3 \pm 3.8)$, expressive communication (9.8 \pm 4.0$)$, and fine motor $(10.2 \pm 2.8)$ domains did not differ significantly from population means $(10 \pm 3)$, whereas
TABLE 2. Near-infrared spectroscopy data

\begin{tabular}{lccc}
\hline & Preoperative & Intraoperative & Postoperative \\
\hline $\mathrm{rSO}_{2}(\%)$ & $71(53-80)$ & - & - \\
$\mathrm{rSO}_{2}$ nadir $(\%)$ & - & $41(33-46)$ & $55(41-61)$ \\
$\mathrm{rSO}_{2} \%$ decrease & - & $36.1(26.6-50)$ & $20.5(1.4-36)$ \\
\hline \multicolumn{3}{c}{ Intraoperative } & Postoperative \\
\hline $\mathrm{rSO}_{2}$ nadir & & \\
$<30 \%$ & $4(12.9 \%)$ & $1(3.2 \%)$ \\
$30 \%-39 \%$ & $9(29 \%)$ & $5(16.1 \%)$ \\
$40 \%-49 \%$ & $12(38.7 \%)$ & $6(19.4 \%)$ \\
$\geq 50 \%$ & $6(19.4 \%)$ & $19(61.3 \%)$ \\
$\mathrm{rSO}_{2} \%$ decrease & & $2(6.5 \%)$ \\
$\geq 50 \%$ & $8(25.8 \%)$ & $5(16.1 \%)$ \\
$40 \%-49 \%$ & $6(19.4 \%)$ & $3(9.7 \%)$ \\
$30 \%-39 \%$ & $8(25.8 \%)$ & $7(22.6 \%)$ \\
$20 \%-29 \%$ & $6(19.4 \%)$ & $14(45.2 \%)$ \\
$<20 \%$ & $3(9.7 \%)$ & \\
\hline$r S O_{2}$, Regional cerebral oxygen level. Reported as median (1Q-3Q) or frequency \\
$($ percentage).
\end{tabular}

the mean score for the gross motor $(8.3 \pm 1.5)$ domain was lower than published norms $(P<.001)$. Five patients $(17 \%)$ were delayed $(>1$ standard deviation below the normative mean) in cognitive development, 7 patients $(23 \%)$ were delayed in receptive communication, 5 patients $(18 \%)$ were delayed in expressive communication, 2 patients $(7 \%)$ were delayed in fine motor development, and 3 patients $(11 \%)$ were delayed in gross motor development.

Table 3 shows the univariable correlations among preoperative, IO, PO, and perioperative NIRS measures and ND scores. The IO percent decrease was less than $20 \%$ in 3 patients who had diagnoses of hypoplastic left heart syndrome $(\mathrm{N}=2)$ and transposition of the great arteries $(\mathrm{N}=1)$ and whose baseline $\mathrm{rSO}_{2}$ were within the first quartile for this sample. A closer look at the data revealed that the small IO percent decrease was likely a function of low baseline $\mathrm{rSO}_{2}$, and that inclusion of patients with less than $20 \%$ decrease in the context of low baseline $\mathrm{rSO}_{2}$ masked the negative relationship between IO percent decrease and language outcomes. After excluding these 3 patients, the correlation between IO percent decrease and receptive communication increased from $r=-0.08$ to $r=-0.44$ and the correlation between IO percent decrease and expressive communication increased from $r=-0.02$ to $r=-0.37$.

\section{Receiver Operating Characteristic Analyses}

ROC analyses evaluated whether preoperative $\mathrm{rSO}_{2}$ could be used to determine the risk of cognitive delay, whether IO percent decrease could be used to determine the risk of receptive communication and expressive communication delays, and whether $\mathrm{PO} \mathrm{rSO}_{2}$ could be used to determine the risk of cognitive and gross motor delays, because these relationships had a significance level of $P$ less than .10 in exploratory correlational analyses. Table E2 
TABLE 3. Univariable correlations between near-infrared spectroscopy data and neurodevelopmental outcomes

\begin{tabular}{|c|c|c|c|c|c|}
\hline & Cognitive & Receptive communication & Expressive communication & Fine motor & Gross motor \\
\hline Preoperative $\mathrm{rSO}_{2}$ & $0.31 \|$ & 0.07 & 0.14 & 0.19 & 0.09 \\
\hline Perioperative $\mathrm{rSO}_{2}$ nadir* & 0.28 & 0.26 & 0.23 & 0.04 & 0.12 \\
\hline $\mathrm{IO} \mathrm{rSO}_{2}$ nadir & 0.21 & 0.29 & 0.28 & -0.01 & 0.03 \\
\hline $\mathrm{PO} \mathrm{rSO}_{2}$ nadir & $0.32 \|$ & 0.06 & -0.03 & 0.18 & $0.37 \|$ \\
\hline Perioperative $\%$ decrease $\dagger, \ddagger$ & -0.29 & $-0.39 \S$ & -0.28 & -0.11 & -0.13 \\
\hline IO $\%$ decrease $\ddagger$ & -0.23 & $-0.44 \S$ & $-0.37 \|$ & -0.09 & -0.08 \\
\hline PO $\%$ decrease & 0.03 & 0.02 & 0.09 & -0.02 & -0.22 \\
\hline
\end{tabular}

$\mathrm{rSO}_{2}$, Regional cerebral oxygen level; $\mathrm{IO}$, intraoperative; $\mathrm{PO}$, postoperative. *Refers to the lowest $\mathrm{rSO}_{2}$ over the preoperative, IO, and PO periods. $\dagger$ Refers to the greatest percent decrease from baseline $\mathrm{rSO}_{2}$ over the $\mathrm{IO}$ and $\mathrm{PO}$ periods. $\ddagger$ Analyses exclude the 3 patients who had less than a $20 \%$ decrease from baseline $\mathrm{rSO} \mathrm{O}_{2}$ during the $\mathrm{IO}$ period. $\S P<.05 . \| P<.10$.

displays sensitivity, specificity, positive predictive value (PPV), and negative predictive value (NPV) estimates for optimal and alternative thresholds.

Preoperative $\mathrm{rSO}_{2}$ was not useful for prediction of cognitive delay (AUC, 0.53). IO percent decrease was moderately useful for prediction of receptive communication delay (AUC, 0.63), but not for prediction of expressive communication delay (AUC, 0.55). The optimal threshold for predicting receptive communication delay was $52 \%$ IO decrease from baseline, which had a sensitivity of $75 \%$, specificity of $83 \%$, PPV of $43 \%$, and NPV of $95 \%$. Patients with IO percent decrease greater than $52 \%(\mathrm{~N}=7)$ had lower receptive communication scores than patients with IO percent decrease less than $52 \%(\mathrm{~N}=21)(t[26]=2.3$, $P=.03)$. Figure 1 shows the scores across all 5 ND domains for patients with an IO percent decrease greater than and less than $52 \%$.

$\mathrm{PO} \mathrm{rSO}_{2}$ nadir was predictive of both cognitive delay (AUC, 0.66) and gross motor delay (AUC, 0.60). The optimal $\mathrm{PO} \mathrm{rSO}_{2}$ nadir threshold for predicting cognitive delay was $56 \%$, which had a sensitivity of $100 \%$, specificity of $56 \%$, PPV of $31 \%$, and NPV of $100 \%$. Patients with a $\mathrm{PO} \mathrm{rSO}_{2}$ nadir less than $56 \%(\mathrm{~N}=16)$ had lower

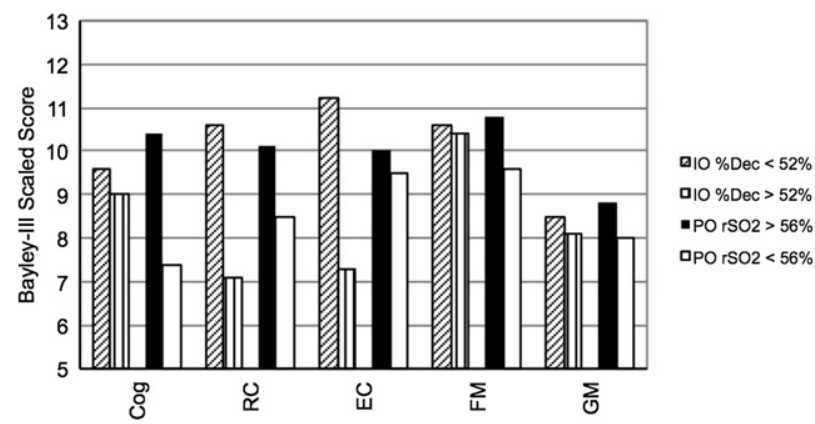

FIGURE 1. ND outcomes of patients with an IO percent decrease from baseline $\mathrm{rSO}_{2}$ greater than and less than $52 \%$ and a $\mathrm{PO} \mathrm{rSO}_{2}$ nadir greater than and less than $56 \%$. Scores have a normative mean of 10 and standard deviation of 3 . IO \% Dec, Intraoperative percent decrease from baseline $\mathrm{rSO}_{2} ; \mathrm{PO} \mathrm{rSO}_{2}$, lowest postoperative regional cerebral oxygen saturation (nadir); $C o g$, cognitive; $R C$, receptive communication; $E C$, expressive communication; $F M$, fine motor; $G M$, gross motor. cognitive scores than patients with a $\mathrm{PO} \mathrm{rSO}_{2}$ nadir $56 \%$ or greater $(\mathrm{N}=14)(t[28]=-2.8, P=.01)$. Figure 1 shows the scores across all 5 ND domains for patients with a PO $\mathrm{rSO}_{2}$ nadir greater than and less than $56 \%$. The optimal $\mathrm{PO} \mathrm{rSO}_{2}$ nadir threshold for predicting gross motor delay was $49 \%$, which had a sensitivity of $67 \%$, specificity of $67 \%$, PPV of $20 \%$, and NPV of $94 \%$. Patients with a PO $\mathrm{rSO}_{2}$ nadir less than $49 \%(\mathrm{~N}=12)$ had lower gross motor scores than patients with a $\mathrm{PO} \mathrm{rSO}_{2}$ nadir $49 \%$ or greater $(\mathrm{N}=19)(t[25]=-2.5, P=.02)$.

\section{Multivariable Linear Regression Models}

Table 4 shows the final multivariable models for each ND outcome.

Cognitive outcome. The final multivariable model for cognitive outcome consisted of length of hospital stay and PO $\mathrm{rSO}_{2}$ nadir less than $56 \%$, which together accounted for $37 \%$ of the variance. Of note, the observed relationship between $\mathrm{PO} \mathrm{rSO}_{2}$ nadir less than $56 \%$ and cognitive outcome appears independent of baseline $\mathrm{rSO}_{2}$, preoperative cyanosis, and $\mathrm{IO} \mathrm{rSO}_{2}$ nadir, because the strength of this relationship ( $r=-0.46$ ) did not meaningfully change when controlling for baseline $\mathrm{rSO}_{2}(r=-0.46)$, preoperative cyanosis $(r=-0.44)$, or ${\mathrm{IO} \mathrm{rSO}_{2}}_{2}$ nadir $(r=-0.43)$, or after

TABLE 4. Final stepwise linear regression models

\begin{tabular}{lccccc}
\hline & $\boldsymbol{B}$ & $\boldsymbol{S E}(\boldsymbol{B})$ & $\boldsymbol{\beta}$ & $\boldsymbol{P}$ & $\boldsymbol{R}^{2}$ \\
\hline Regression 1: cognitive & & & & & .37 \\
$\quad$ Length of hospital stay & -0.04 & 0.02 & -.44 & .009 & \\
$\quad$ PO rSO 2 nadir $<56 \%$ & -2.5 & 1.0 & -.39 & .02 & \\
Regression 2: RC* & & & & & .13 \\
$\quad$ IO \% decrease $>$ 52\% & -3.4 & 1.5 & -.40 & .03 & \\
Regression 3: fine motor & & & & & .23 \\
$\quad$ Length of CICU stay & -0.05 & 0.02 & -.51 & .007 & \\
Regression 4: gross motor & & & & & .46 \\
$\quad$ Multiple DHCA & -1.8 & 0.45 & -.59 & .001 & \\
$\quad$ Parent education level & 1.3 & 0.43 & .44 & .006 & \\
\hline
\end{tabular}

$S E$, Standard error; $P O$, postoperative; $r \mathrm{SO}_{2}$, regional cerebral oxygen level; $R C$, receptive communication; $I O$, intraoperative; $C I C U$, cardiac intensive care unit; $D H C A$, deep hypothermic circulatory arrest. *Analyses exclude the 3 patients who had less than a $20 \%$ decrease from baseline $\mathrm{rSO}_{2}$ during the intraoperative period. 
excluding patients with baseline $\mathrm{rSO}_{2}$ less than $40 \%$ $(r=-0.43)$. Neither single ventricle physiology nor duration of DHCA entered into the final stepwise regression model. When duration of DHCA was forced into the model, the added predictive value of $\mathrm{PO} \mathrm{rSO}_{2}$ nadir less than $56 \%$ remained marginally significant $\left(\Delta R^{2}=0.11, P=.052\right)$. When single ventricle physiology was forced into the model, the added predictive value of $\mathrm{PO} \mathrm{rSO}_{2}$ nadir less than $56 \%$ did not reach statistical significance $\left(\Delta R^{2}=0.08, P=.11\right)$.

Language outcomes. The final multivariable model for receptive communication outcome consisted of IO percent decrease greater than $52 \%$, which accounted for $13 \%$ of the variance. No clinical or sociodemographic characteristics were associated with expressive communication outcome.

Motor outcomes. The final multivariable model for fine motor outcome included length of cardiac intensive care unit stay, which accounted for $23 \%$ of the variance. The final multivariable model for gross motor outcome consisted of education level of accompanying parent and multiple procedures with DHCA, which together accounted for $46 \%$ of the variance.

\section{DISCUSSION}

Because of dramatic improvements in survival after surgical repair of CHD, identification of strategies to improve ND outcome has become the next challenge. NIRS monitoring has emerged as a surrogate means of evaluating $\mathrm{rSO}_{2}$ and has been introduced into clinical practice in the absence of empirical and reproducible data relating NIRS values to ND outcome. The limited research to date has focused exclusively on $\mathrm{rSO}_{2}$ during the preoperative or intraoperative periods, despite studies documenting decreased oxygen delivery during the early PO period ${ }^{12-15}$ and an association between PO events and neurologic or ND outcomes. ${ }^{16-18}$

In this cohort, the relative IO decrease from baseline $\mathrm{rSO}_{2}$ was associated with receptive communication outcome, whereas the $\mathrm{rSO}_{2}$ nadir during the $\mathrm{PO}$ period was associated with cognitive outcome. This suggests that perioperative NIRS monitoring may enhance our ability to predict ND outcomes beyond what is possible when considering other clinical characteristics or monitoring methods. These results also suggest that the specific NIRS measures associated with ND outcome differ across the continuum of the perioperative period. Percent decrease from baseline $\mathrm{rSO}_{2}$ had greater predictive value for ND outcome during the IO period; this may reflect the fact that the baseline was established at the beginning of the procedure after induction of anesthesia, and the lowest values were commonly reached in association with important changes in perfusion, allowing for a broader range of change during the IO period. Our results also suggest that the specific ND domain associated with decreased $\mathrm{rSO}_{2}$ may differ across the perioperative period. Although this may be a function of limited statistical power given the small sample size, this could also indicate that different areas of the brain exhibit different thresholds to hypoxic injury.

Results from the IO period are generally consistent with our prior work, which revealed a relationship between $\mathrm{rSO}_{2}$ and receptive communication, but not other ND domains. ${ }^{11}$ It is interesting that ${\mathrm{IO} \mathrm{rSO}_{2}}_{2}$ seems to have a more robust relationship with receptive, compared with expressive, communication. It is possible that performance on this domain reflects not only language development but also attentional abilities, known to be affected among children with complex CHD. ${ }^{22}$ Poor focus or impulsivity could negatively affect a child's performance on receptive communication tasks, which require the child to attend to the examiner's verbal cues, to determine which pictures or objects correspond with each verbal cue, and to follow directions. Kussman and colleagues ${ }^{9}$ found an association between IO $\mathrm{rSO}_{2}$ and the Psychomotor Development Index, but not the Mental Development Index, of the second edition of the Bayley Scales in a cohort of patients undergoing biventricular repair. However, the study by Kussman and colleagues differed from the current investigation with regard to the cardiac diagnoses of the sample, need for additional cardiac surgeries before testing, ND testing battery, and age at which children were tested, making cross-study comparisons difficult.

The current study extends prior work on the assessment of $\mathrm{rSO}_{2}$ by including $\mathrm{rSO}_{2}$ values during the early $\mathrm{PO}$ period, therefore evaluating the clinical utility of PO NIRS monitoring to predict ND outcomes while considering important clinical variables. The NIRS threshold with the best balance of sensitivity and specificity was an IO percent decrease from baseline $\mathrm{rSO}_{2}$ of $52 \%$, which correctly classified $75 \%$ of children with receptive communication delay and $87 \%$ of children without delay. A patient whose IO $\mathrm{rSO}_{2}$ remains within $52 \%$ of the baseline value has a $95 \%$ chance of experiencing normal receptive communication development on the basis of this patient cohort. A PO $\mathrm{rSO}_{2}$ nadir threshold of $56 \%$ correctly classified $100 \%$ of children with cognitive delay, and a patient whose $\mathrm{PO}$ $\mathrm{rSO}_{2}$ nadir is at or greater than $56 \%$ has a $100 \%$ chance of experiencing normal cognitive development on the basis of this cohort. However, the specificity of this measure for cognitive delay was not sufficiently better than chance. On the basis of these results, perioperative NIRS data could be used in clinical practice to predict which children are at highest risk for delay and in need of early intervention and which are likely to exhibit typical development. However, these data must be validated in a prospective cohort. In addition, prospective studies are necessary to evaluate whether NIRS can be used to guide perioperative management decisions and, more important, to improve ND outcome. Without these data, the clinical utility of perioperative NIRS is likely to remain a topic of controversy. 
In the past, optimal NIRS measurements have not been defined. Thresholds for high risk have been assigned in the absence of empirical studies directly examining their relationship to ND outcome. ${ }^{6}$ We used an empirical method to determine optimal thresholds, and the differences between these thresholds and those previously suggested to be associated with increased risk are notable. The identified IO percent decrease from baseline $\mathrm{rSO}_{2}$ threshold of $52 \%$ is larger than the previously suggested decrease of $20 \%{ }^{6}$ Because nearly all patients experienced at least a $20 \%$ decrease from baseline in the IO period, a 20\% threshold lacks specificity. It should also be noted that in this cohort, the 3 patients who exhibited less than a $20 \%$ decrease from baseline during the $\mathrm{IO}$ period had low baseline $\mathrm{rSO}_{2}$ and exhibited significant ND impairments. Therefore, caution should be exercised when considering percent decrease as an isolated measure. The $\mathrm{PO} \mathrm{rSO}_{2}$ nadir threshold of $56 \%$ is more conservative than the previously recommended value of $40 \%{ }^{6}$ Perhaps this is a reflection of the different conditions patients encounter during the PO period, including normothermia and increased activity, which are likely to affect the metabolic rate of oxygen in the brain.

Because of the variability regarding the most predictive $\mathrm{rSO}_{2}$ measure and the most affected ND domain between the IO and PO periods, it was not possible to determine if and how the optimal threshold for a single $\mathrm{rSO}_{2}$ measure would change over the perioperative period. However, the pattern of results suggests that optimal thresholds for IO $\mathrm{rSO}_{2}$ may be higher than for $\mathrm{PO} \mathrm{rSO}_{2}$. This may be related to the neuroprotection conferred by the use of hypothermia during the $\mathrm{IO}$ period. It should also be noted that $\mathrm{rSO}_{2}$ nadir and percent decrease from baseline $\mathrm{rSO}_{2}$ showed weaker relationships to ND outcome when the entire continuum of the perioperative period was considered, compared with when the IO and PO periods were considered independent of one another. These data suggest that emphasis on one single NIRS measure or threshold across the continuum of the perioperative period may not be clinically useful.

Of note and consistent with prior research, ${ }^{11,23}$ the length of cardiac intensive care unit and total hospital stay remained a robust predictor of neurodevelopment, even when other patient- and procedure-related variables were considered. Infants who experience a prolonged hospital stay are likely sicker, are prone to complications, and may be missing important experiences and opportunities that would promote their early development. The observed relationship between parent education level and neurodevelopment is consistent with previously documented relationships between socioeconomic status measures and neurodevelopment. ${ }^{24}$ This is likely driven by multiple factors, including access to financial and educational resources and underlying genetic differences that contribute to both parent academic achievement and early childhood development.

\section{Study Limitations}

Limitations of this study include its small sample size and cross-sectional retrospective design, which precludes conclusions regarding causality. This article reports on secondary analyses of patients enrolled across 2 research studies examining ND outcomes after infant cardiac surgery. Patients included in these analyses underwent more complex procedures with longer support times and greater use of DHCA when compared with the broader population of infants undergoing cardiac surgery. These findings may not generalize to patients who do not have DHCA. It should also be noted that half of the participants in this study had multiple surgeries and hospitalizations; therefore, although the primary focus of this study was on the first surgery with CPB during which patients received perioperative NIRS monitoring, it is possible that events occurring during other perioperative periods affected ND outcomes. Last, because PO NIRS values were entered into the medical record on an hourly basis, cumulative minutes at or below a particular $\mathrm{rSO}_{2}$ could not be calculated and brief decreases in $\mathrm{rSO}_{2}$ may not be captured in the available data.

\section{CONCLUSIONS}

NIRS monitoring during and beyond the IO period seems to enhance the ability to predict ND outcomes at 2 years of age. Specific NIRS measures associated with ND outcome and the optimal thresholds on these measures differ across the continuum of the perioperative period. The use of a single NIRS measure or threshold across the continuum of the perioperative period may not be appropriate. Further research is needed to investigate these relationships in a prospective manner and to examine whether goal-directed therapy can alter NIRS measurements and long-term ND outcomes.

\section{References}

1. Wernovsky G. Current insights regarding neurological and developmental abnormalities in children and young adults with complex congenital cardiac disease. Cardiol Young. 2006;16(1 Suppl):92-104.

2. Daubeney PEF, Smith DC, Pilkington SN, Janke E, Gareth AC, Smith DC, et al. Cerebral oxygenation during paediatric cardiac surgery: identification of vulnerable periods using near infrared spectroscopy. Eur J Cardiothoracic Surg. 1998; 13:370-7

3. Tweddell JS, Ghanayem NS, Hoffman GM. Pro: NIRS is "standard of care" for postoperative management. Semin Thorac Cardiovasc Surg Pediatr Card Surg Аnпu. 2010;13:44-50.

4. Edmonds HL. Pro: all cardiac surgical patients should have intraoperative cerebral oxygenation monitoring. J Cardiothorac Vasc Anesth. 2006;20:445-9.

5. Hoffman GM. Neurologic monitoring on cardiopulmonary bypass: what are we obligated to do? Ann Thorac Surg. 2006;81:S2373-80.

6. Hoffman GM. Pro: near-infrared spectroscopy should be used for all cardiopulmonary bypass. J Cardiothorac Vasc Anesth. 2006;20:606-12.

7. Davies LK, Janelle GM. Con: all cardiac surgical patients should not have intraoperative cerebral oxygenation monitoring. J Cardiothorac Vasc Anesth. 2006 20:450-5.

8. Hirsch JC, Charpie JR, Ohye RG, Gurney JG. Near-infrared spectroscopy: what we know and what we need to know - a systematic review of the congenital heart disease literature. J Thorac Cardiovasc Surg. 2009;137:154-9. 
9. Kussman BD, Wypij D, Laussen PC, Soul JS, Bellinger DC, DiNardo JA, et al. Relationship of intraoperative cerebral oxygen saturation to neurodevelopmental outcome and brain magnetic resonance imaging at 1 year of age in infants undergoing biventricular repair. Circulation. 2010;122:245-54.

10. Toet MC, Flinterman A, van de Laar I, Vries JW, Bennink GB, Uiterwaal CS, et al. Cerebral oxygen saturation and electrical brain activity before, during, and up to 36 hours after arterial switch procedure in neonates without preexisting brain damage: Its relationship to neurodevelopmental outcome. Exp Brain Res. 2005;165:343-50.

11. Simons J, Sood E, Derby CD, Pizarro C. Predictive value of near-infrared spectroscopy on neurodevelopmental outcome after surgery for congenital heart disease in infancy. $J$ Thorac Cardiovasc Surg. 2012;143:118-25.

12. Hoffman TM, Wernovsky G, Atz AM, Kulik TJ, Nelson DP, Chang AC, et al. Efficacy and safety of milrinone in preventing low cardiac output syndrome in infants and children after corrective surgery for congenital heart disease. Circulation. 2003;107:996-1002.

13. Wernovsky G, Wypij D, Jonas RA, Mayer JE Jr, Hanley FL, Hickey PR, et al. Postoperative course and hemodynamic profile after the arterial switch operation in neonates and infants-a comparison of low-flow cardiopulmonary bypass and circulatory arrest. Circulation. 1995;92:2226-35.

14. Tweddell JS, Hoffman GM. Postoperative management in patients with complex congenital heart disease. Semin Thorac Cardiovasc Surg Pediatr Card Surg Апnи. 2002;5:187-205.

15. Parr GVS, Blackstone EH, Kirklin JW. Cardiac performance and mortality early after intracardiac surgery in infants and young children. Circulation. 1975;51: 867-74.

16. Hoffman GM, Mussatto KA, Brosig CL, Ghanayem NS, Musa N, Fedderly RT, et al. Systemic venous oxygen saturation after the Norwood procedure and childhood neurodevelopmental outcome. J Thorac Cardiovasc Surg. 2005;130: 1094-100.

17. Rappaport LA, Wypij D, Bellinger DC, Helmers SL, Holmes GL, Barnes PD, et al. Relation of seizures after cardiac surgery in early infancy to neurodevelopmental outcome. Circulation. 1998;97:773-9.

18. Bellinger DC, Jonas RA, Rappaport LA, Wypij D, Wernovsky G, Kuban KC, et al. Developmental and neurologic status of children after heart surgery with hypothermic circulatory arrest or low-flow cardiopulmonary bypass. $N$ Engl $J$ Med. 1995;332:549-55.

19. Sood E, Simons J, Davies R, Pizarro C. Neurodevelopmental outcomes following infant cardiac surgery: Can intermittent perfusion extend the safe duration of deep hypothermic circulatory arrest? Abstract presented at the 38th Annual Meeting of the Western Thoracic Surgical Association, Maui, Hawaii, June 2012.

20. Bayley N. Bayley Scales of Infant Development. Third ed. San Antonio, TX: Harcourt Assessment, Inc; 2006.

21. Kraemer HC, Morgan GA, Leech NL, Gliner JA, Vaske JJ, Harmon RJ. Measures of clinical significance. J Am Acad Child Adolesc Psychiatry. 2003;42:1524-9.

22. Shillingford AJ, Glanzman MM, Ittenbach RF, Clancy RR, Gaynor JW, Wernovsky G. Inattention, hyperactivity, and school performance in a population of school-age children with complex congenital heart disease. Pediatrics. 2008; 121:e759-67.

23. Newburger J, Wypij D, Bellinger D, du Plessis AJ, Kuban KC, Rappaport LA, et al. Length of stay after infant heart surgery is related to cognitive outcome at age 8 years. J Pediatr. 2003;143:67-73.

24. Newburger JW, Sleeper LA, Bellinger DC, Goldberg CS, Tabbutt S, Lu M, et al. Early developmental outcome in children with hypoplastic left heart syndrome and related anomalies: the Single Ventricle Reconstruction trial. Circulation. 2012;125:2081-91.

\section{Discussion}

Dr Charles Fraser (Houston, Tex). I congratulate Dr Sood and colleagues from Nemours Cardiac Center for this body of work adding further information to this ongoing and sometimes curious debate about the benefits of NIRS in providing relevant perioperative physiologic monitoring and potentially optimizing ND outcomes in children with critical cardiac disease undergoing surgery.

Without attempting to resummarize this well-written manuscript and elegant presentation that we've just heard, I synopsize this work as follows: In a heterogeneous group of 31 children undergoing repair in infancy, 8 of whom had single ventricles, using various CPB strategies but a lot of circulatory arrest, you've observed and concluded that perioperative NIRS monitoring has predictive utility in ultimate ND outcome. Specific observations included noting that a $52 \%$ decrease from baseline IO NIRS saturation correctly classified $75 \%$ of children with receptive communication delay and that a PO threshold of $56 \%$ correctly classified $100 \%$ of children with cognitive delay.

As the authors probably know, these data are somewhat consistent with ours from Texas Children's Hospital as recently presented at the Society of Thoracic Surgeons meeting in Florida by my colleague Dean Andropoulos. We presented there and will soon report in print our findings that low perioperative cerebral cortical oxygen saturations as measured by NIRS has a direct relationship with 12-month Bayley developmental scores. To our view, and apparently now yours, measuring oxygen delivery to the brain has clinical relevance in predicting outcome.

You note that $94 \%$ of the children had circulatory arrest. Other than the isolated measured data points documenting the lowest IO saturations, were you able to measure the total time spent at nadir? We should probably ask the same questions about preoperative and PO levels.

Dr Sood. We did not measure total time spent at nadir. This study examined NIRS data collected using the prior software, which did not report AUC. Also, we obtained PO NIRS data from the medical record, which were entered on an hourly basis. However, we examined total time spent below specific NIRS thresholds in our prior study of IO NIRS and did not find an association with ND outcomes. Kussman and colleagues ${ }^{9}$ examined the relationship between IO NIRS data and ND outcomes at 1 year of age and did not find an association between AUC for $\mathrm{rSO}_{2}$ less than or equal to $45 \%$ and ND outcomes. But certainly this needs to be further examined in future studies.

Dr Fraser. Well, that's a good segue into question 2. Your group has published 3 related abstracts on this subject in the last 4 months. In the January issue of the Journal you concluded, and I quote: "In a contemporary cohort of infants undergoing surgery for congenital heart disease, neurodevelopmental outcomes at 2 years are largely influenced by patient-related characteristics. Although receptive communication appears to be influenced by the NIRS nadir, the predictive value remains unclear."

Elsewhere in this meeting's program, there is a poster abstract in which you conclude, apparently in the same group of patients, that "duration of hypothermic circulatory arrest was not predictive of any neurodevelopmental outcome."

Finally, in this article you conclude, "NIRS monitoring during and beyond the IO period seems to enhance the ability to predict ND outcomes at 2 years of age." So please help me tie this all together.

Dr Sood. Our recent article in the Journal reported on only IO data, and the results were generally consistent with what was found in the present study. The prior study found a relationship between IO NIRS nadir and receptive communication outcome, and the present study found a relationship between IO percent decrease from baseline and receptive communication outcome. The relationship that we previously described between IO NIRS nadir and receptive communication did not reach statistical significance 
in the present sample. However, receptive communication was the domain associated with NIRS data in both studies.

Although the study samples for the 3 abstracts that you referred to do overlap, they are not equivalent samples. Additional patients have been tested since the 2 prior abstracts, and the specific inclusion criteria differ across studies. For example, several patients included in the IO NIRS study were excluded from this study because perioperative NIRS data were not available.

Dr Christian Pizarro (Wilmington, Del). Maybe I could help with that answer. I think that last year when we presented some of these data at the meeting in Philadelphia, the question was raised to the fact that there were no PO data, and obviously things could occur in that immediate period, particularly in the first 48 hours, and that was the stimulus for us to go back and try to obtain and look at the PO data and NIRS monitoring.

Now, the challenge was that not every patient had PO NIRS data available, and therefore that's why there is a slight difference in which patients were included in each study. But the idea for this particular project was to look at all patients who had NIRS data available before, during, and after surgery.

Dr Fraser. Christian might want to comment on this as well. In the last sentence of your conclusion in the article you state that "Further research is needed to investigate these relationships in a prospective manner and to examine whether goal-directed therapy can alter NIRS measurements and long-term ND outcomes."

As we talked about earlier, first, you can alter NIRS measurements with direct interventions (eg, higher hematocrit, higher flow rates). On the basis of what you've learned so far, do you plan to change your strategy going forward and act on the NIRS information rather than just observe it?

Dr Pizarro. That's a great question. Part of the challenge is the fact that when it comes to IO interventions, obviously the intuitive mode of treatment would be to alter perfusion, for example, use regional antegrade cerebral perfusion; however, we do not know the true impact of this strategy that may increase cerebral edema, for example. This is different than increasing the hematocrit in the intensive care unit or giving inotropes and trying to augment cardiac output by other means. So as stated before, CPB is probably an unnatural state, and there is some potential morbidity from it.

If we look at the Michigan study where randomization of regional cerebral perfusion and circulatory arrest was undertaken, actually no difference was demonstrated. Although we could alter the number that the NIRS monitor gives us, the consequences and the impact of the measures that we used to achieve that might not necessarily improve the overall outcome. What we discovered through this analysis is that it seems like the cutoff, of $40 \%$ as a nadir, might be too low, and data would suggest that perhaps the cutoff is in the 50s. We would be inclined to continue to explore this, enroll more patients to include in the analysis, and probably, if the data sustain the study's findings, modify our approach to it.

Dr J. William Gaynor (Philadelphia, Pa). I agree with your final statement. We have to be careful about using historical controls. This is an important first step, along with the data from Texas Children's, that we're beginning to get data that may correlate some of these measurements at certain time points, and particularly in the PO period, with ND outcomes. These type of data will be important, although it take some time before it becomes completely ingrained in clinical practice.

We recently did a systematic review of the literature for many of the things that we do for neuroprotection and neuromonitoring, and the level of evidence for what we do is appalling. There is almost nothing that reaches class 1 evidence. We have an opportunity if people will be willing to design appropriate trials, based on this type of evidence, to begin to look at NIRS in a formal prospective way with evaluation of goal-directed therapy.

Some of the work by Dr Licht has suggested that increasing delivery may not be the best thing. Perhaps we have to decrease demand, for example, keeping patients intubated or sedated longer, perhaps cooling a little bit. I don't know. If we begin to have some idea of the balance between oxygen use and oxygen delivery, then we should design a trial, so going forward we actually have evidence for some of the things we do. 
TABLE E1. Clinical and sociodemographic characteristics and near-infrared spectroscopy measures considered for entry into stepwise linear regression models

\begin{tabular}{|c|c|c|c|c|c|}
\hline & \multicolumn{5}{|c|}{ Univariable correlations with ND outcome } \\
\hline & $r$ & $\boldsymbol{P}$ & & $r$ & $\boldsymbol{P}$ \\
\hline \multicolumn{3}{|l|}{ Cognitive } & \multicolumn{3}{|l|}{ Fine motor } \\
\hline Single ventricle physiology* & -0.40 & .03 & Parent education level & 0.40 & .04 \\
\hline Significant comorbidity* & -0.41 & .02 & Length of CICU stay & -0.51 & .007 \\
\hline Duration of DHCA & -0.39 & .03 & Length of hospital stay & -0.47 & .01 \\
\hline $\mathrm{PO} \mathrm{rSO}_{2}$ nadir $<56 \%$ & -0.46 & .01 & \multicolumn{3}{|l|}{ Gross motor } \\
\hline Length of CICU stay & -0.46 & .01 & Parent education level & 0.39 & .047 \\
\hline Length of hospital stay & -0.52 & .003 & Significant comorbidity & -0.43 & .03 \\
\hline Multiple DHCA* & -0.41 & .02 & Preoperative cyanosis* & -0.45 & .02 \\
\hline \multicolumn{3}{|l|}{ Receptive communication } & Duration of DHCA & -0.44 & .02 \\
\hline 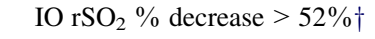 & -0.40 & .03 & $\mathrm{PO} \mathrm{rSO}_{2}$ nadir $<49 \%$ & -0.46 & .01 \\
\hline \multicolumn{3}{|l|}{ Expressive communication } & Length of CICU stay & -0.46 & .02 \\
\hline \multirow[t]{3}{*}{ None } & & & Length of hospital stay & -0.45 & .02 \\
\hline & & & Multiple DHCA & -0.55 & .003 \\
\hline & & & Cumulative DHCA & -0.44 & .02 \\
\hline
\end{tabular}

$\overline{N D}$, Neurodevelopmental; $\mathrm{DHCA}$, deep hypothermic circulatory arrest; $\mathrm{PO}$, postoperative; $r \mathrm{SO}_{2}$, regional cerebral oxygen level; $\mathrm{CICU}$, cardiac intensive care unit; $\mathrm{IO}$, intraoperative. *Dichotomous variables were coded as present (1) or absent (0). †Analyses exclude the 3 patients who had less than a $20 \%$ decrease from baseline to intraoperative $\mathrm{rSO}_{2}$ nadir.

TABLE E2. Clinical utility estimates for intraoperative and postoperative near-infrared spectroscopy thresholds

\begin{tabular}{|c|c|c|c|c|c|c|}
\hline ND domain/NIRS measure & AUC & Threshold & Sensitivity & Specificity & PPV & NPV \\
\hline \multicolumn{7}{|l|}{ Receptive communication } \\
\hline \multirow[t]{3}{*}{ IO \% decrease* } & 0.63 & 49.7 & 0.75 & 0.79 & 0.38 & 0.95 \\
\hline & & 52.2 & 0.75 & 0.83 & 0.43 & 0.95 \\
\hline & & 54.6 & 0.50 & 0.83 & 0.33 & 0.91 \\
\hline \multicolumn{7}{|l|}{ Cognitive } \\
\hline \multirow[t]{3}{*}{ 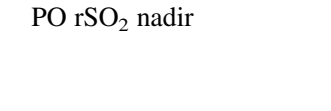 } & 0.66 & 57 & 1.00 & 0.48 & 0.28 & 1.00 \\
\hline & & 55.5 & 1.00 & 0.56 & 0.31 & 1.00 \\
\hline & & 53 & 0.80 & 0.60 & 0.29 & 0.94 \\
\hline \multicolumn{7}{|l|}{ Gross motor } \\
\hline \multirow[t]{3}{*}{$\mathrm{PO} \mathrm{rSO}_{2}$ nadir } & 0.60 & 52.5 & 0.67 & 0.58 & 0.17 & 0.93 \\
\hline & & 48.5 & 0.67 & 0.67 & 0.20 & 0.94 \\
\hline & & 43.5 & 0.33 & 0.67 & 0.11 & 0.89 \\
\hline
\end{tabular}

Bold text signifies optimal thresholds based on the balance of sensitivity, specificity, positive predictive value, and negative predictive value. $N D$, Neurodevelopmental; $N I R S$, near-infrared spectroscopy; $A U C$, area under the curve; $\mathrm{PPV}$, positive predictive value; $\mathrm{NPV}$, negative predictive value; $I O$, intraoperative; $P O$, postoperative; $r \mathrm{SO}_{2}$, regional cerebral oxygen level. *Analyses exclude the 3 patients who had less than a $20 \%$ decrease from baseline to intraoperative $\mathrm{rSO}_{2}$ nadir. 